20

\title{
Сапфировый нейрохирургический зонд для аспирации опухолей мозга с демаркацией границ с помощью спектроскопии*
}

\author{
(с) И.А. Шикунова ${ }^{1}$, И.Н. Долганова ${ }^{1,2}$, Г.М. Катыба ${ }^{1,2,3}$, К.И. Зайцев ${ }^{2,3}$, В.Н. Курлов ${ }^{1, \text { ॠ }}$ \\ ${ }^{1}$ Институт фризики твердого тела Российской академии наук, \\ 142432 Черноголовка, Россия \\ ${ }^{2}$ Московский государственный технический университет им. Н.Э. Баумана, \\ 105005 Москва, Россия \\ ${ }^{3}$ Институт общей физики им. А.М. Прохорова Российской академии наук, \\ 119991 Москва, Россия \\ ฯ e-mail: kurlov@issp.ac.ru
}

Поступила в редакцию 03.12.2018 г.

В окончательной редакции 16.01.2019 г.

Принята к публикации 31.01.2019 г.

\begin{abstract}
Разработан контактный сапфировый нейрохирургический зонд для удаления опухоли головного мозга с возможностью интраоперационной флуоресцентной диагностики и лазерной коагуляции прилегающих кровеносных сосудов. Оптимизирована геометрия сапфирового нейрозонда для повышения чувствительности флуоресцентной диагностики. Для этого проведена серия вычислительных экспериментов с помощью метода Монте-Карло. Разработана методика выращивания профилированного кристалла сапфира переменного сечения, с помощью которой изготовлен пилотный образец сапфирового нейрозонда. Проведена его экспериментальная апробация.
\end{abstract}

DOI: $10.21883 /$ OS.2019.05.47663.12-19

\section{Введение}

Лечение мультиформной глиобластомы, относящейся к злокачественным первичным опухолям головного мозга, является одной из самых серьезных проблем в нейроонкологии из-за ее инфильтративного характера роста и молекулярных механизмов защиты клеток новообразования при различных видах терапии [1-3]. Несмотря на успехи в разработке современного оборудования для предоперационного и интраоперационного контроля, а также заметные достижения в области иммунной, лучевой и химиотерапии прогноз для пациентов с глиобластомами остается крайне неутешительным [4-8]. Медиана выживаемости с момента постановки диагноза обычно составляет около одного года; 5-летняя выживаемость не превышает 4-7\%, и даже в самых благоприятных ситуациях большинство пациентов умирает в течение двух лет [8-10].

Современные подходы к лечению сочетают резекцию опухоли с последующей адьювантной терапией, причем общая выживаемость пациентов с глиобластомами напрямую зависит от удаления опухоли с максимальной радикальностью при минимально возможном повреждении окружающей мозговой ткани, что определяет продолжительность и качество дальнейшей жизни пациента [11-14]. Тотальное удаление без развития или усугуб-

\footnotetext{
* The 22nd Annual Conference Saratov Fall Meeting 2018 (SFM'18): VI International Symposium „Optics and Biophotonics“ and XXII International School for Junior Scientists and Students on Optics, Laser Physics \& Biophotonics, September 24-29, 2018, Saratov, Russia. https://www.sgu.ru/structure/fiz/saratov-fall-meeting/previousconferences/sara
}

ления неврологического дефицита является сложной и актуальной проблемой из-за отсутствия четких границ между опухолевой тканью и мозговым веществом при инфильтративном росте опухоли, особенно при ее локализации в функционально значимых областях мозга [15].

Крайне важным фактором, влияющим на максимально возможное хирургическое удаление опухоли, является возможность определения границ новообразования с использованием интраоперационной визуализации и спектроскопии. Существуют различные принципы диагностики, позволяющие решить проблему интраоперационной демаркации опухолевой ткани и нормальной ткани мозга, такие как спектроскопия комбинационного рассеяния (рамановская спектроскопия) [16,17], оптическая когерентная томография (ОКТ) [18], интраоперационная компьютерная и магнитно-резонансная томография [19], ультразвуковое сканирование и трехмерная безрамная ультразвуковая нейронавигация [20-22], электрофизиологическое картирование и электростимуляция $[23,24]$, мультимодальная навигация [25], визуализация с помощью ИК камер [26], терагерцовая импульсная спектроскопия и визуализация [27], а также различные комбинации этих методов.

Одним из эффективных методов интраоперационной навигации, позволяющим визуализировать границу между опухолью и веществом мозга, а также идентифицировать участки активно пролиферирующей опухолевой ткани, является интраоперационная флуоресцентная спектроскопия и визуализация на базе эндогенной (аутофлуоресценция) [28,29] или экзогенной [30] флуоресценции. Экзогенные флуорофоры (фотосенсибилиза- 
торы) накапливаются в опухолях головного мозга в высоких концентрациях. Таким образом возможно быстрое интраоперационное обнаружение и визуализация злокачественных глиом, отмеченных различными флуоресцентными биомаркерами [31]. В настоящее время в качестве стандарта в хирургии глиом высокой степени злокачественности в соответствии с клиническими рекомендациями по использованию интраоперационной флуоресцентной диагностики в хирургии опухолей головного мозга применяется 5-аминолевулиновая кислота (5-АЛК) [14,32-38]. Метод флуоресцентной диагностики целесообразно использовать для объема резекции поверхностной части опухоли с учетом функционально значимых зон мозга [14].

На сегодняшний день наибольшее распространение получил интраоперационный флуоресцентный имиджинг, который существенно повышает тотальность удаления опухоли [39]. Регистрируемое в реальном времени флуоресцентное видеоизображение дает значительные преимущества при удалении глиальных опухолей. Высокое накопление в мозге веществ, использующихся для флуоресцентной демаркации (в частности, протопорфирина IX (PpIX) в присутствии 5-АЛК), обусловлено повреждением гематоэнцефалического барьера в клетках опухоли, при этом здоровые клетки их не накапливают. Это приводит к необычно высокому контрасту флуоресценции (до 40:1), регистрируемой в злокачественной ткани по отношению к флуоресценции нормальной ткани. Однако на различных стадиях операции, особенно после удаления основного объема опухоли, планарная качественная картина флуоресценции при использовании современных микроскопов и эндоскопов с флуоресцентными фильтрами позволяет проводить лишь качественную оценку видеофлуоресценции и не является достаточной для проведения полного удаления. Требуется проведение количественных измерений методами оптической спектроскопии накопления PpIX в тканях $[37,38,40]$.

Для количественного анализа флуоресценции применяются оптоволоконные спектрометрические зонды, позволяющие с высокой точностью обнаруживать фрагменты опухоли. Они допускают измерение флуоресценции с погруженным в ткань зондом, что увеличивает глубину диагностики [41]. Для увеличения точности диагностики применяется анализ нескольких показателей (комбинированная спектроскопия), таких как накопление 5-АЛК-индуцированного РpIX, светорассеяние, оксигенация и кровенаполнение $[42,43]$.

Несмотря на относительно высокую эффективность 5-АЛК при флуоресцентной диагностике глиом высокой степени злокачественности (WHOgrade III, IV), применение этого метода для диагностики глиом низкой степени злокачественности (WHOgrade I, II) далеко не всегда возможно - лишь 10-20\% подобных опухолей демонстрируют видимое накопление PpIX [44]. Представляется перспективным применение новых методов терагерцовой спектроскопии и визуализации тканей для

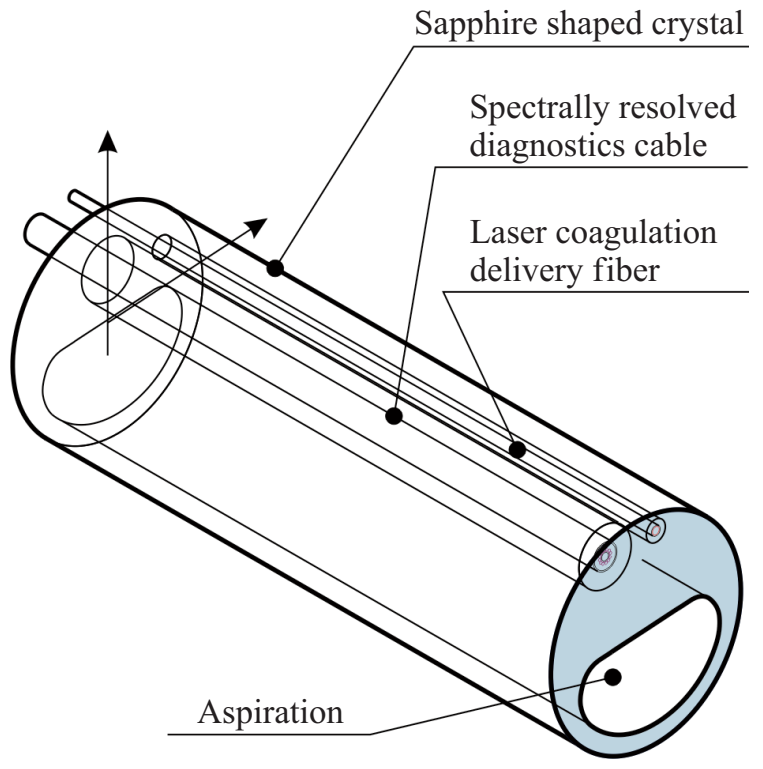

Рис. 1. Схема контактной части сапфирового нейрозонда с совмещением в одном инструменте аспирации тканей, экзогенной флуоресцентной диагностики и лазерной коагуляции.

диагностики глиом головного мозга всех степеней злокачественности (в том числе и WHOgrade I, II) [45]. Однако методы терагерцовой диагностики по-прежнему далеки от клинической практики, так как терагерцовое оборудование до сих пор остается громоздким и дорогостоящим.

Существующие точечные зонды для измерений оптических характеристик тканей мозга с целью диагностики являются, как правило, неконтактными из-за невозможности стерилизации инструментов, содержащих оптические волокна. Это существенно ограничивает их применение в ходе операции, особенно после удаления основного объема опухоли, когда требуется диагностика глубинно расположенных опухолей со сложной конфигурацией ложа, а операционная зона представляет собой полость с нависающими краями. На практике они применимы на начальной стадии для уточнения границ опухоли in situ и дополняют видеофлуоресцентный канал при операции на открытом мозге. Кроме того, использование различных инструментов для интраоперационного картирования тканей и удаления выявленных участков опухоли может приводить к ошибкам позиционирования инструментов, удалению здоровой ткани и пропускам очагов опухоли. Актуальнейшей задачей нейрохирургии на сегодняшний день является повышение точности и тотальности удаления опухолей за счет совмещения функций диагностики и удаления в одном нейрохирургическом инструменте.

В настоящей работе представлен новый нейрохирургический контактный зонд на основе профилированного кристалла сапфира, предназначенный для интраоперационного определения границ опухоли, ее последующего удаления (аспирации), а также коагуля- 

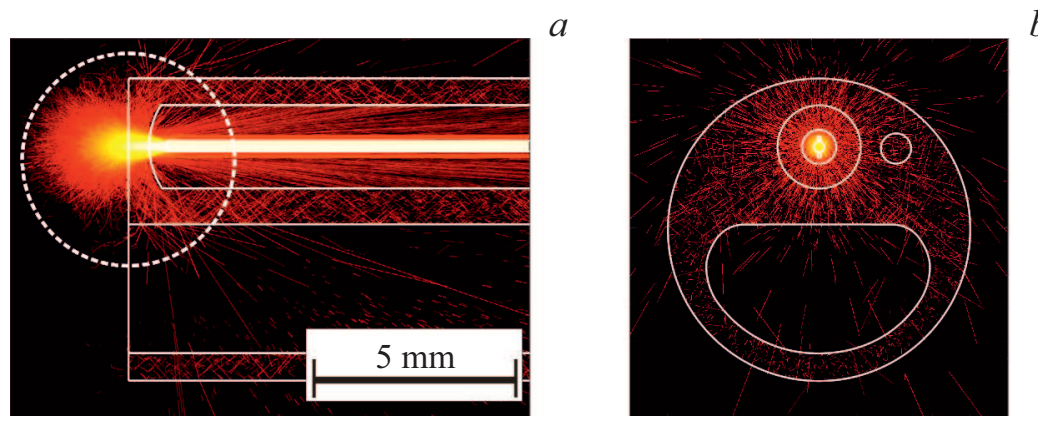

$b$
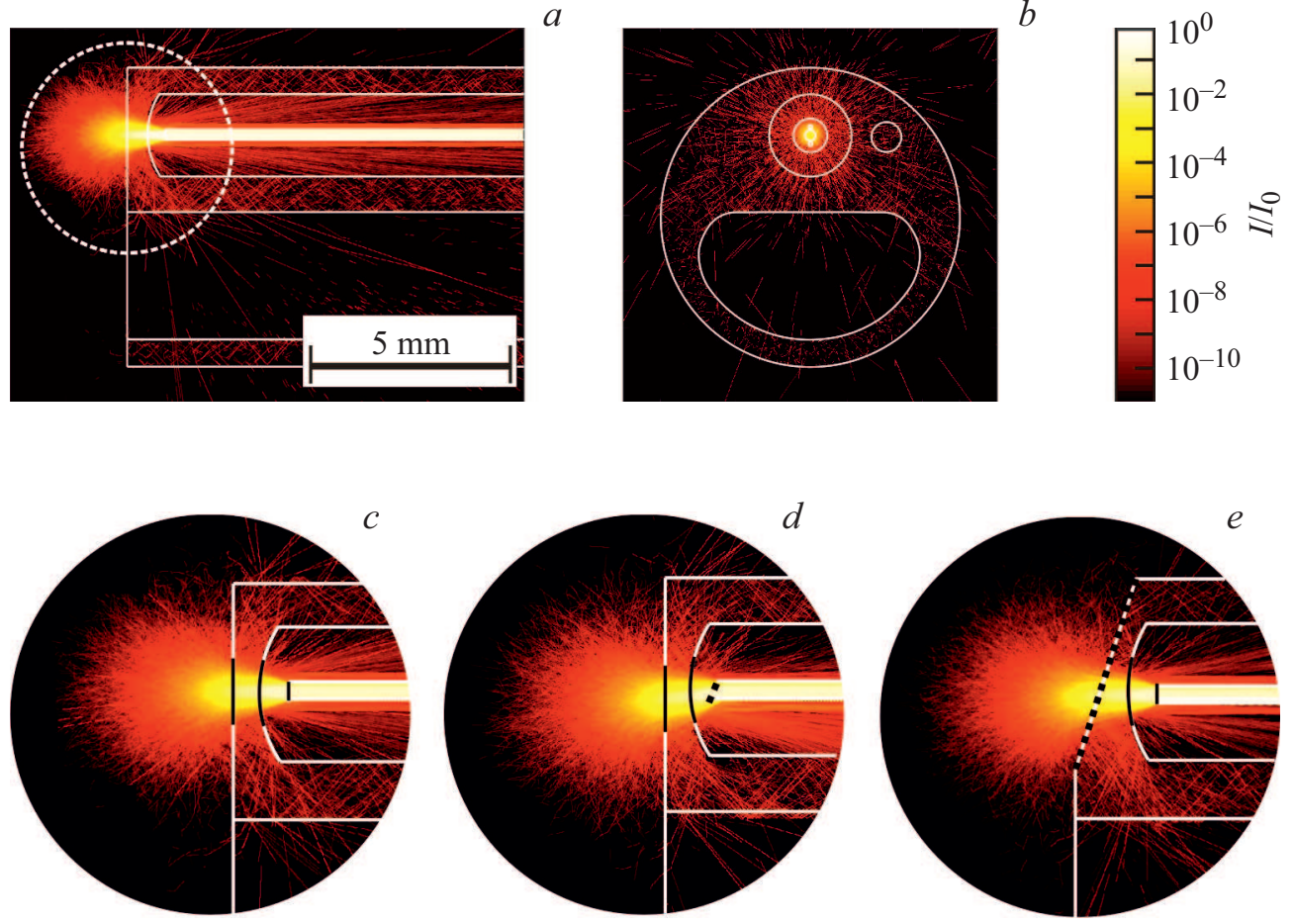

Рис. 2. Распределение плотности мощности лазерного излучения в ткани мозга и в объеме сапфирового зонда с внешним диаметром $8 \mathrm{~mm}$ (моделирование методом Монте-Карло): $a, b$ - торцы зонда и световода перпендикулярны оси зонда; $c$ увеличенное изображение $a ; d-$ выходной торец световода имеет наклон $15^{\circ} ; e$ - торец зонда имеет наклонную грань под углом $15^{\circ}$ к оси световода.

ции прилегающих сосудов. Выбор сапфира в качестве материала нейрохирургического зонда обусловлен его высокой механической прочностью, теплопроводностью, термостойкостью, химической инертностью к крови и тканям человека, в том числе электролитической пассивностью, коррозионной стойкостью, биосовместимостью и пропусканием в широком оптическом диапазоне [46]. Сочетание уникальных свойств сапфира с достоинствами метода выращивания профилированных кристаллов сапфира (возможность получения кристаллов заданной формы без дополнительной трудоемкой механической обработки, высокая точность поддержания геометрии поперечного сечения, оптическое качество поверхностей) [47-51] позволяет успешно решить проблему создания новых высокотехнологичных инструментов для хирургии, флуоресцентной диагностики, криодеструкции, фотодинамической терапии, лазерной коагуляции [52-60]. Сапфир является немагнитным материалом, что делает возможным его использование в качестве основы хирургического инструментария для операций, проводимых под контролем МРТ, что чрезвычайно актуально для нейрохирургии опухолей головного мозга [61].

Схема предложенного сапфирового контактного нейрозонда для удаления опухоли головного мозга показана на рис. 1. Кроме сквозного продольного аспирационного канала, через который фрагменты опухоли и кровь удаляются из операционной зоны, в сапфировом стержне располагаются закрытые с рабочего торца каналы для размещения в них оптического волоконного зонда для флуоресцентной диагностики и кварцевого световода для доставки лазерного излучения с целью коагуляции и гемостаза.

\section{Оптимизация геометрии сапфирового зонда}

Для оптимизации геометрии контактной части сапфирового зонда и определения его чувствительности при флуоресцентной диагностике был разработан ряд упрощенных математических моделей распространения оптического излучения для системы „световод-сапфировый нейрозонд-биоткань““. Зона чувствительности зонда расположена в контактирующей с зондом ткани, по оси световода и занимает объем, равный нескольким кубическим миллиметрам. Для улучшения пространственного совмещения зоны диагностики и зоны аспирации можно использовать наклонные грани, сформированные на рабочем торце зонда, либо световод, имеющий скошенный рабочий торец или миниатюрную призму на конце.

На рис. 2 показаны результаты моделирования методом Монте-Карло распространения излучения, доставляемого волокном в канале сапфирового нейрозонда для трех случаев. Наклонные грани влияют на величину доли излучения, рассеянного в зонде при внутреннем отражении. Данное излучение (засветка), которое может 
ухудшать как пороговую чувствительность при диагностике, так и разрешаемый контраст (пространственная чувствительность), имеет минимальное значение в случае существования наклонной грани на торце зонда.

При контактной флуоресцентной диагностике глубина залегания содержащих флуорофор объектов, которые могут быть обнаружены волоконным световодом, не превышает нескольких миллиметров. Это связано с сильным градиентом плотности мощности в ткани при внешнем точечном облучении. Излучение флуоресценции в свою очередь ослабляется в слое ткани на пути к приемному волокну. При переходе от закрытого канала к монолитной части непосредственно в процессе выращивания кристалла формируется дно с кривизной. Таким образом, перед волокнами существует дополнительный оптический элемент - отрицательная линза, имеющая как большую кривизну вогнутой поверхности, так и высокий показатель преломления. Это оказывает влияние на формируемые световые поля в ткани, а также вводит угловую селекцию лучей в приемном канале, что приводит к стягиванию объема диагностики и улучшению пространственной чувствительности диагностики.

Методом Монте-Карло определялась оптимальная толщина слоя сапфира по оптической оси зонда для получения максимальной эффективности захвата диагностического излучения, для которой в дальнейшем оценивали пространственную чувствительность и другие параметры, необходимые для работы инструментом. Моделирование показало, что с увеличением толщины слоя сапфира по оси световода происходит снижение эффективности захвата флуоресценции (рис. 3). Исходя из технологических возможностей формирования рабочего торца зонда, толщина слоя сапфира должна быть уменьшена до нескольких долей миллиметра. При этом малый объем кристалла, где создается высокая плотность излучения, не должен содержать каких- либо включений, приводящих к рассеянию излучения. Выбранное значение толщины сапфира по оси световода в рабочей части нейрозонда составило $0.5 \mathrm{~mm}$. Также высокие требования предъявляются к качеству поверхности дна канала, содержащего световод.

Пространственное разрешение флуоресцентной диагностики зависит от размера и взаимного положения волокон в применяемом световоде. В качестве параметров световода для осуществления этих типов диагностики были взяты параметры зонда Reflex12/1(300), в котором 12 осветительных волокон диаметром $100 \mu$ m окружают центральное волокно в металлической оболочке диаметром $300 \mu \mathrm{m}$ (числовая апертура $N A=0.22$, „Optofiber“, Москва) [62].

Оценка пространственного разрешения флуоресцентной диагностики проводилась путем моделирования поперечного смещения точечных объектов с флуорофором (сферы диаметром $0.2 \mathrm{~mm}$ ) относительно сапфирового зонда с размещенным световодом с измерением

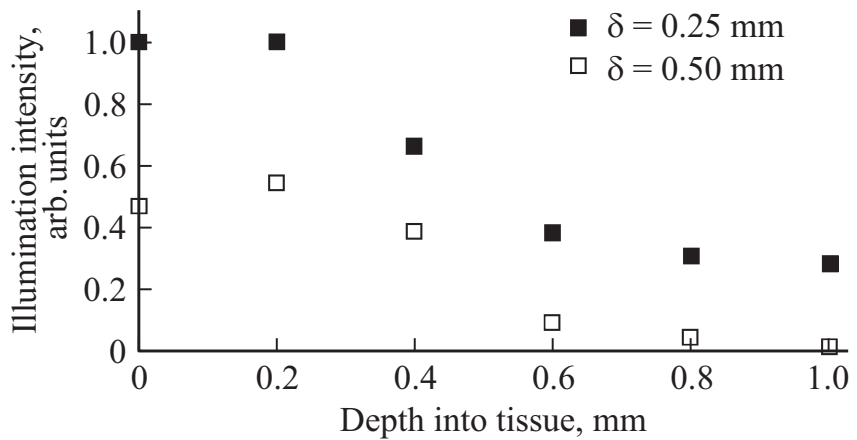

Рис. 3. Освещенность на приемном волокне, создаваемая микросферой, находящейся в ткани на различной глубине, для зонда с различной толщиной слоя сапфира по оси световода $\delta$.

доли излучения, захватываемого центральным приемным волокном. Моделирование показало, что зонд может быть использован для различения двух точечных источников флуоресценции, разнесенных на расстояние $1.6 \mathrm{~mm}$

\section{Выращивание из расплава многоканального сапфирового нейрозонда}

На рис. 4 показана схема выращивания из расплава сапфирового многоканального стержня требуемой геометрии, которую практически невозможно получить механической обработкой из-за высокой твердости сапфира. Сечение кристалла определяется конструкцией формообразователя, при этом размеры кристалла могут варьироваться лишь в узком диапазоне, определяемом формой мениска расплава $[63,64]$. Для получения стержня переходного сечения основание мениска расплава отрывается от кромок одной части формообразователя, задающего первоначальную форму (все три канала открыты; рис. 4,a), и зацепляется за кромки другой части формообразователя, задающего следующую форму поперечного сечения (один канал открыт, вместо двух других каналов растет монолитная часть; рис. 4, b). При этом также регулируется масса расплава, поступающая к фронту кристаллизации, путем изменения положения формообразователя относительно уровня расплава в тигле, температуры и скорости выращивания [55]. Таким образом, выращивание кристаллов с изменяемой по длине формой поперечного сечения можно рассматривать как чередование стационарного роста с переходным режимом кристаллизации. При таком переходе наружный мениск расплава остается фиксированным на наружных кромках формообразователя, а внутренние мениски расплава частично исключаются.

Для контроля состояния фронта кристаллизации и предотвращения возникновения дефектов, связанных с переохлаждением, в стационарном и переходных процессах разработана автоматическая система управления для 


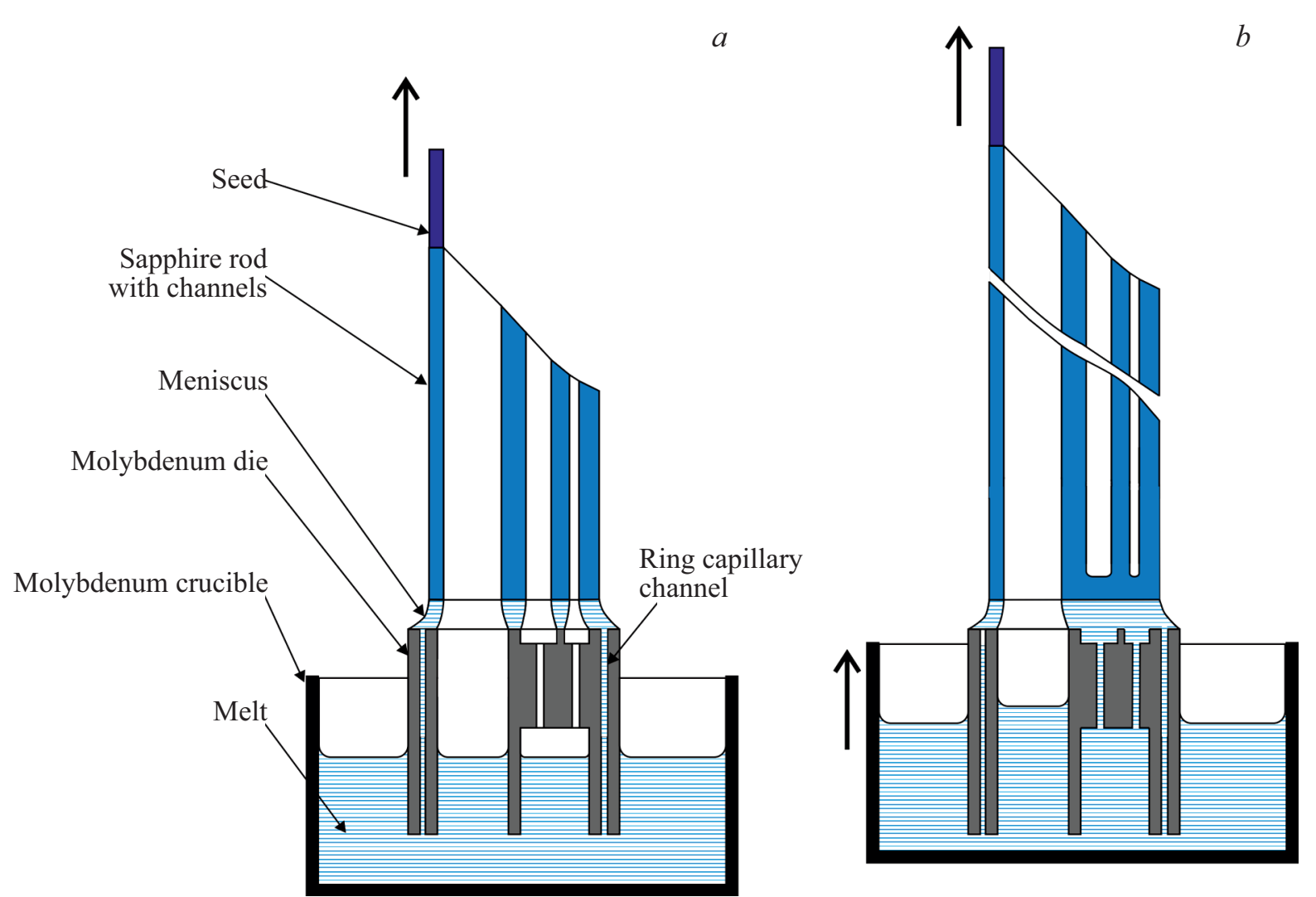

Рис. 4. Схема выращивания сапфирового стержня: $(a)$ с тремя каналами, $(b)$ после перехода на стержень с одним каналом.
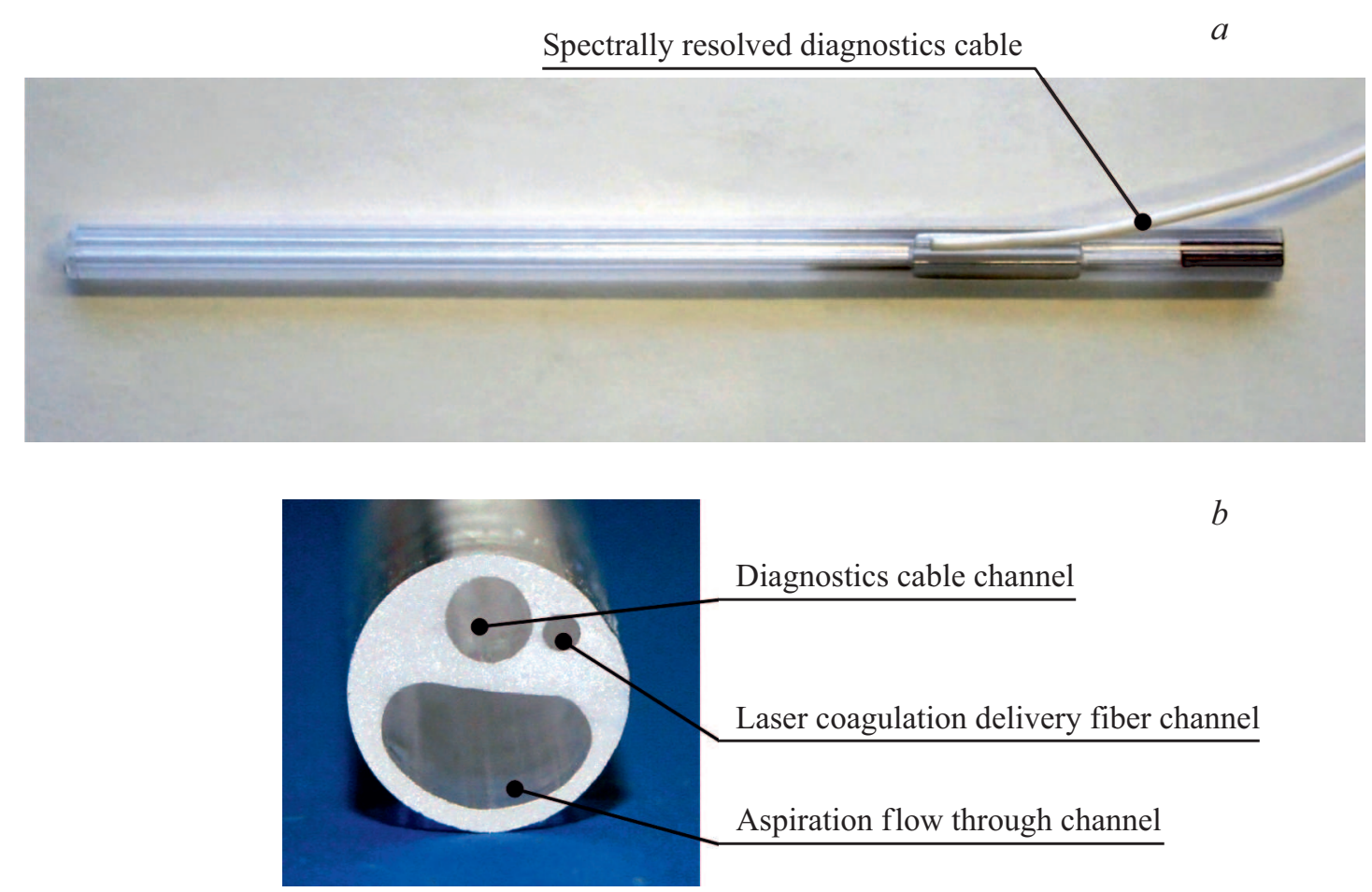

Рис. 5. Сапфировый контактный нейрозонд диаметром $8 \mathrm{~mm}$ с возможностью диагностики, коагуляции и аспирации: $a-$ фотография зонда, $b-$ фотография поперечного сечения зонда. 


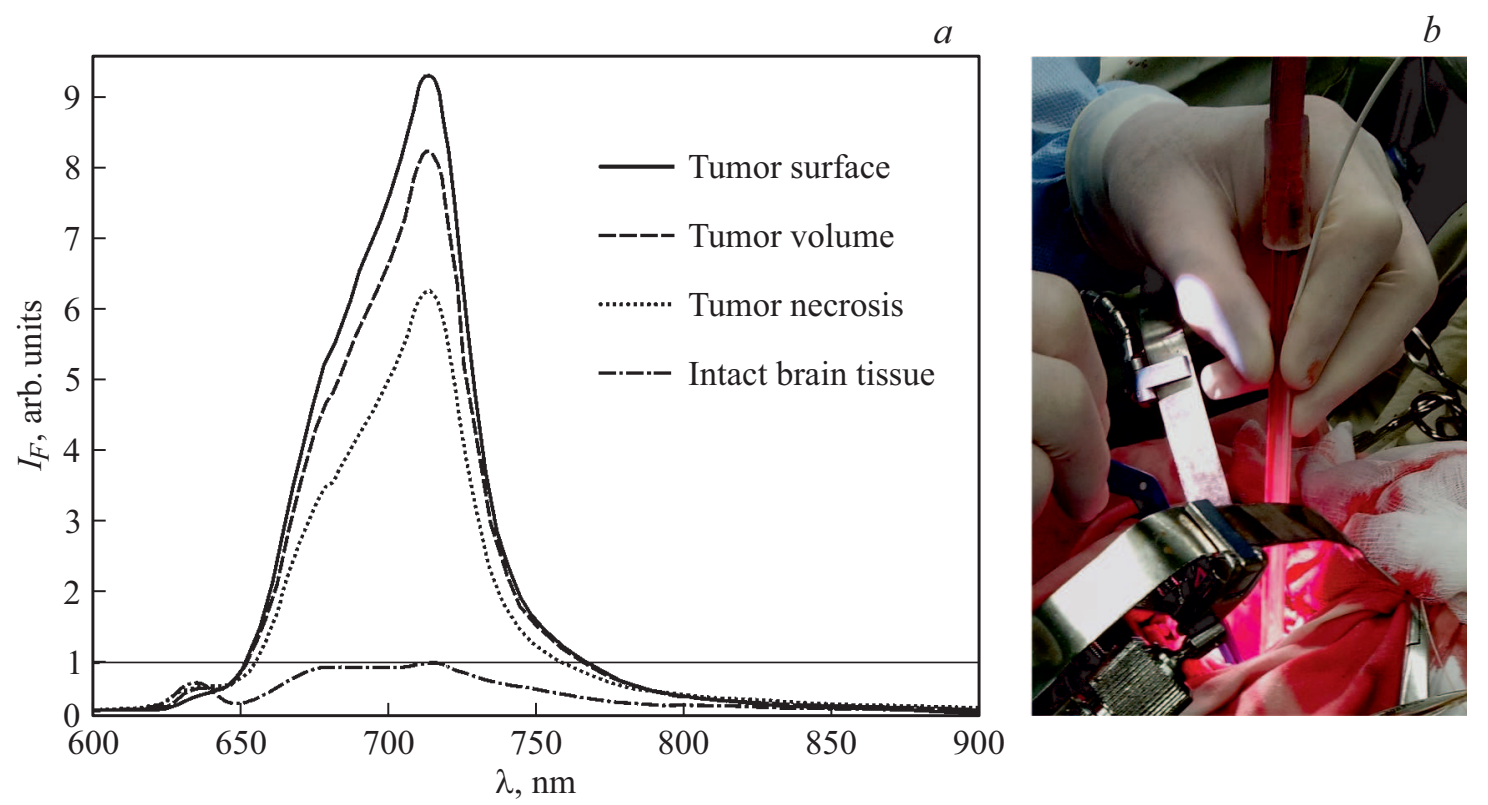

Рис. 6. Результаты апробации нейрозонда: (a) регистрируемые спектры флуоресценции для области здоровой ткани, края опухоли и опухолевой ткани, нормированные к максимуму флуоресценции здоровой ткани мозга; $(b)$ фотография апробации сапфирового зонда в клинических условиях (нейрохирургическое отделение МОНИКИ им. М.Ф. Владимирского).

выращивания профилированных кристаллов с использованием датчика веса. Принципы системы управления и реализация контроля состояния фронта кристаллизации в процессе выращивания подробно описаны в [65]. В качестве основного параметра, по которому осуществлялось управление состоянием фронта кристаллизации, использовалась амплитуда колебаний отклонения реальной скорости изменения массы от программной. Именно на анализе амплитуды колебаний и ее корректировке соответственным изменением мощности нагрева основано управление формой и качеством растущего кристалла.

Выращивание сапфировых многоканальных стержней переменного сечения проводилось на установке индукционного нагрева, снабженной датчиком веса кристалла с чувствительностью $20 \mathrm{mg}$, в защитной атмосфере аргона под давлением $1.1-1.3$ at, направление выращивание - вдоль оси $\{0001\}$. Формообразующее устройство, тигель и система экранов были изготовлены из молибдена. Выращивание проводилось в очень узком диапазоне скоростей вытягивания при малой высоте мениска, что обеспечивало состояние фронта кристаллизации, близкое к переохлажденному.

На рис. 5 показан сапфировый нейрозонд, который может быть использован как в режиме операций на открытом мозге, так и для нейроэндоскопических операций с применением троакаров с инструментальным каналом [66]. Волокна вводятся через боковую проточку зонда, удаленную от рабочего конца инструмента и закрывающуюся стерильным рукавом так, что в прямом контакте с биотканью находится только сапфировый нейрозонд. Система компонуется из следующих основных узлов: сапфировый многоканальный зонд, аспиратор, $Y$-образный световод спектрометрической системы и спектрофотометр со средством обработки и отображения информации, источники излучения для возбуждения флуоресценции и коагуляции с целью гемостаза.

\section{Апробация сапфирового нейрозонда}

Зонд был использован в процессе стандартной нейрохирургической операции (нейрохирургическое отделение МОНИКИ им. М.Ф. Владимирского) для идентификации в режиме реального времени инвазивных опухолей мозга и их удаления, в том числе для зондирования ложа опухоли и максимального удаления ее фрагментов без повреждения важных структур, что позволило увеличить послеоперационную безрецидивную 6-месячную выживаемость [55]. Для флуоресцентной диагностики использовался препарат Аласенс (НИОПИК, Россия) из расчета $20 \mathrm{mg} / \mathrm{kg}$. На основании спектрометрической картины на всех этапах удаления опухоли проводилась биопсия опухолевой ткани, зоны ее некроза, пограничной зоны и интактного мозга, выявлены средние значения их спектральных характеристик, таких как безразмерный индекс флуоресценции (ИФ) и отношение интенсивности флуоресценции $\mathrm{PpIX}$ в диапазоне 690-730 nm к интенсивности рассеянного лазерного излучения, в том числе in vitro. Диагностическим критерием выступает величина флуоресцентного контраста, представляющая из себя отношение ИФ опухолевой ткани к ИФ интактной ткани мозга (рис. 6). ИФ в среднем для опухоли составил $12 \pm 5$, для зоны некроза - 
$4.5 \pm 2$, для пограничной зоны $-5 \pm 1.5$, для мозга $1.5 \pm 1$.

\section{Выводы}

Разработан портативный контактный зонд для удаления опухолей головного мозга. С помощью сапфирового зонда, содержащего волокна спектрометрической системы, производится локальная флуоресцентная диагностика для выявления участков опухоли. Помимо диагностики злокачественности клеток зонд позволяет использовать в случае необходимости коагуляцию для гемостаза, фотодинамическую терапию и лазерную термотерапию. Использование сапфирового наконечника с интеграцией в нем функций одномоментной флуоронавигации и удаления опухоли оптимизирует точность и радикальность операции. Проведена оптимизация геометрии сапфирового нейрозонда для повышения чувствительности флуоресцентной диагностики, что позволяет более точно определять границы опухоли непосредственно в процессе ее удаления.

\section{Финансирование работы}

Работа выполнена при частичной поддержке гранта РФФИ № 18-08-01230.

\section{Соблюдение этических стандартов}

Все процедуры, выполненные в исследовании с участием людей, соответствуют этическим стандартам институционального и/или национального комитета по исследовательской этике и Хельсинкской декларации 1964 г. и ее последующим изменениям или сопоставимым нормам этики. От каждого из включенных в исследование участников было получено информированное добровольное согласие.

\section{Список литературы}

[1] Wen P.Y., Kesari S. // N. Engl. J. Med. 2008. V. 359. N 5. P. 492. doi 10.1056/NEJMra0708126

[2] Adamson C., Kanu O., Mehta A. Di C., Lin N., Mattox A.K., Bigner D.D. // Expert Opin. Investig. Drugs. 2009. V. 18 (8). P. 1061-1083. doi 10.1517/13543780903052764

[3] Ramirez Y., Weatherbee J.L., Wheelhouse R.T., Ross A.H. // Pharmaceuticals. 2013. V. 6. N 12. P. 1475. doi 10.3390/ph6121475

[4] Robins H.I., Chang S., Butowski N., Mehta M. // Curr. Oncol. Rep. 2007. V. 9. P. 66. doi 10.1007/BF02951428

[5] Stupp R., Mason W.P., van den Bent M.J., Weller M., Fisher B., Taphoorn M.J.B., Belanger K., Brandes A.A., Marosi C., Bogdahn U., Curschmann J., Janzer R.C., Ludwin S.K., Gorlia T., Allgeier A., Lacombe D., Cairncross J.G., Eisenhauer E., Mirimanoff R.O. // N. Engl. J. Med. 2005. V. 352. P. 987. doi 10.1056/NEJMoa043330
[6] Safdie F., Brandhorst S., Wei M., Wang W., Lee C., Hwang S., Conti P.S., Chen T.C., Longo V.D. // PLoS One. 2012. V. 7. N 9. P. e44603. doi 10.1371/journal.pone.0044603

[7] Minniti G., Muni R., Lanzetta G., Marchetti P., Enrici R.M. // Anticancer Res. 2009. V. 29. N 12. P. 5171.

[8] Anton K., Baehring J.M., Mayer T. // Hematol. Oncol. Clin. North. Am. 2012. V. 26. N 4. P. 825. doi 10.1016/j.hoc.2012.04.006

[9] Lamborn K.R., Chang S.M., Prados M.D. // Neuro Oncol. 2004. V. 6. N 3. P. 227. doi $10.1215 / \mathrm{S} 1152851703000620$

[10] Ostrom Q.T., Gittleman H., Truitt G., Boscia A., Kruchko C., Barnholtz-Sloan J.S. // Neuro-Oncology. 2018. V. 20. N 4. P. iv1. doi 10.1093/neuonc/noy131

[11] Stummer W., Tonn J.C., Mehdorn H.M., Nestler U., Franz K., Goetz C., Bink A., Pichlmeier U. // J. Neurosurg. 2011. V. 114. N 3. P. 613-623. doi 10.3171/2010.3.JNS097

[12] Sanai N., Berger M.S. // Neurosurgery. 2008. V. 62. N 4. P. 753. doi 10.1227/01.neu.0000318159.21731.cf

[13] Schucht P., Beck J., Abu-Isa J., Andereggen L., Murek M., Seidel K., Stieglitz L., Raabe A. // Neurosurgery. 2012. V. 71. N 5. P. 927. doi 10.1227/NEU.0b013e31826d1e6b

[14] Потапов А.А., Горяйнов С.А., Охлопков В.А., Пицхелаури Д.И., Кобяков Г.Л., Жуков В.Ю., Гольбин Д.А., Свистов Д.В., Мартынов Б.В., Кривошапкин А.Л., Гайтан А.С., Анохина Ю.Е., Варюхина М.Д., Гольдберг М.Ф., Кондрашов А.В., Чумакова А.П. // Вопросы нейрохирургии. Н.Н. Бурденко. 2015. № 5. С. 91. doi 10.17116/neiro201579591-101

[15] Smith J.S., Chang E.F., Lamborn K.R., Chang S.M., Prados M.D., Cha S., Tihan T., Vandenberg S., McDermott M.W., Berger M.S. // J. Clin. Oncol. 2008. V. 26. N 8. P. 1338. doi 10.1200/JCO.2007.13.9337

[16] Jermyn M., Mok K., Mercier J., Desroches J., Pichette J., Saint-Arnaud K., Bernstein L., Guiot M.C., Petrecca K., Leblond F. // Sci. Transl. Med. 2015. V. 7. N 274. P. 274 ra19. doi 10.1126/scitranslmed.aaa2384

[17] Cordero E., Latka I., Matthäus C., Schie I., Popp J. // J. Biomed. Opt. 2018. V. 23. N 7. P. 1. doi 10.1117/1.JBO.23.7.071210

[18] Kut C., Chaichana K.L., Xi J., Raza S.M., Ye X., McVeigh E.R., Rodriguez F.J., Quiñones-Hinojosa A., Li X. // Sci. Transl. Med. 2015. V. 7. P. 292ra100. doi 10.1126/scitranslmed.3010611

[19] Black P.M., Moriarty T., Alexander E., Stieg P., Woodard E.J., Gleason P.L., Martin C.H., Kikinis R., Schwartz R.B., Jolesz F.A. // Neurosurg. 1997. V. 41. N 4. P. 831.

[20] Prada F., DelBene M., Mattei L., Casali C., Filippini A., Legnani F., Mangraviti A., Saladino A., Perin A., Richetta C., Vetrano I., Moiraghi A., Saini M., DiMeco F. // J. Ultrasound. 2014. V. 17. N 3. P. 243. doi 10.1007/s40477-014-0111-8

[21] Парфенов В.Е., Свистов Д.В., Савелло А.В., Лапшин Р.А., Цибиров А.В. // Российская нейрохирургия. 2004. № 2. C. 18.

[22] Unsgaard G., Rygh O.M., Selbekk T., Müller T.B., Kolstad F., Lindseth F., Hernes T.A. // Acta Neurochir. 2006. V. 148. N 3. P. 235. doi 10.1007/s00701-005-0688-y

[23] Duffau H., Capelle L., Sichez N., Denvil D., Lopes M., Sichez J.P., Bitar A., Fohanno D. // Brain. 2002. V. 125. P. 199.

[24] Kombos T., Süss O., Vajkoczy P. // Neurosurg. Focus. 2009. V. 27. N 4. P. E5. doi 10.3171/2009.8.FOCUS09140 
[25] González-Darder J.M., González-López P., Talamantes F., Quilis V., Cortés V., García-March G., Roldán P. // Neurosurg. Focus. 2010. V. 28. N 2. P. E5. doi 10.3171/2009.11.FOCUS09234

[26] Kateb B., Yamamoto V., Yu C., Grundfest W., Gruen J.P. // NeuroImage. 2009. V. 47. Suppl. 2. P. T154. doi 10.1016/j.neuroimage.2009.03.043

[27] Ji Y., Oh S., Kang S.-G., Heo J., Kim S.-H., Choi Y., Song S., Son H., Kim S., Lee J., Haam S., Huh Y., Chang J., Joo C., Suh J.-S. // Sci. Rep. 2016. V. 6. P. 36040. doi $10.1038 /$ srep36040

[28] Monici M. // Biotechnol. Annu. Rev. 2005. V. 11. P. 227. doi 10.1016/S1387-2656(05)11007-2

[29] Croce A., Fiorani S., Locatelli D., Nano R., Ceroni M., Tancioni F., Giombelli E., Benericetti E., Bottiroli G. // Photochem. Photobiol. 2015. V. 77. N 3. P. 309. doi 10.1562/0031-8655(2003)077<0309:DPOAFA > 2.0.CO;2

[30] Olivo M., Wilson B. // Intern. J. Oncol. 2004. V. 25. N 1. P. 37. doi 10.3892/ijo.25.1.37

[31] Senders J., Muskens I., Schnoor R., Karhade A., Cote D., Smith T., Broekman M. // ActaNeurochir. 2017. V. 159. P. 151. doi 10.1007/s00701-016-3028-5

[32] Schucht P., Beck J., Abu-Isa J., Andereggen L., Murek M., Seidel K., Stieglitz L., Raabe A. // Neurosurgery. 2012. V. 71. N 5. P. 927-936. doi 10.1227/NEU.0b013e31826d1e6b

[33] Stummer W., Tonn J.C., Mehdorn H.M. Nestler U., Franz K., Goetz C., Bink A., Pichlmeier U. // J. Neurosurg. 2011. V. 114. N 3. P. 613-623. doi 10.3171/2010.3.JNS097

[34] Acerbi F., Cavallo C., Broggi M., Cordella R., Anghileri E., Eoli M., Schiariti M., Broggi G., Ferroli P. // Neurosurg. Rev. 2014. V. 37. N 4. P. 547. doi 10.1007/s10143-014-0546-6

[35] Zhao S., Wu J., Wang C., Liu H., Dong X., Shi C., Shi C., Liu Y., Teng L., Han D., Chen X., Yan, G., Wang L., Shen C., Li H. // PLoS One. 2013. V. 8. N 5. P. e63682. doi 10.1371/journal.pone.0063682

[36] Roberts D.W., Valdes P.A., Harris B.T., Fontaine K.M., Hartov A., Fan X., Ji S., Lollis S.S., Pogue B.W., Leblond F., Tosteson T.D., Wilson B.C., Paulsen K.D. // J. Neurosurg. 2011. V. 113. N 2. P. 595. doi: $10.3171 / 2010.2 . J N S 091322$

[37] Valdes P.A., Leblond F., Kim A. Harris B.T., Wilson B.C., Fan X., Tosteson T.D., Hartov A., Ji S., Erkmen K., Simmons N.E., Paulsen K.D., Roberts D.W. // J. Neurosurg. 2011. V. 115. N 1. P. 11. doi 10.3171/2011.2.JNS101451

[38] Valdes P.A., Bekelis K., Harris B.T., Wilson B.C., Leblond F., Kim A., Simmons N.E., Erkmen K., Paulsen K.D., Roberts D.W. // Neurosurg. 2014. V. 10. P. 74. doi 10.1227/NEU.0000000000000117

[39] Hefti M., Mehdorn H.M., Albert I., Dörner L. // Curr. Med. Imag. Rev. 2010. V. 6. N 4. P. 254. doi 10.2174/157340510793205503

[40] Pogue B.W., Gibbs-Strauss S., Valdés P.A., Samkoe K., David $W$., Roberts D.W., Paulsen K.D. // IEEE J. Sel. Top. Quantum Electron. 2010. V. 16. P. 493. doi 10.1109/JSTQE.2009.2034541

[41] Kuroiwa T., Kajimoto Y., Miyatake S.I., Miyashita M. // Minimally Invasive Neurosurgery and Multidisciplinary Neurotraumatology. / Ed. by Kanno T., Kato Y. Tokyo: Springer, 2006. P. 80. doi 10.1007/4-431-28576-8_12

[42] Потапов А.А., Горяйнов А.А., Лощенов В.Б., Савельева Т.А., Гаврилов А.Г., Охлопков В.А., Жуков В.Ю., Зеленков П.В., Гольбин Д.А., Шурхай В.А., Шишкина Л.В.,
Грачев П.В., Холодиява М.Н., Кузьмин С.Г., Ворожцов Г.Н., Чумакова А.П. // Вопросы нейрохирургииим. Н.Н. Бурденко. 2013. Т. 77. № 1. С. 3.

[43] Савельева Т.А., Лощенов В.Б., Горяйнов С.А., Шишкина Л.В., Потапов А.А. // Российский химический журн. 2013. T. 57. № 5. C. 39.

[44] Jaber M., Wolfer J., Ewelt C., Holling M., Hasselblatt M., Niederstadt T., Zoubi T., Weckesser M., Stummer W. // Neurosurgery. 2016. V. 78. P. 401. doi 10.1227/NEU.0000000000001020

[45] Gavdush A.A., Chernomyrdin N.V., Malakhov K.M., Beshplav S.-I.T., Dolganova I.N., Kosyrkova A.V., Nikitin P.V., Musina G.R., Katyba G.M., Reshetov I.V., Cherkasova O.P., Komandin G.A., Karasik V.E., Potapov A.A., Tuchin V.V., Zaytsev K.I. // J. Biomed. Opt. 2019. V. 24. Iss. 2. P. 027001. doi 10.1117/1.JBO.24.2.027001

[46] Kurlov V.N. // Reference Module in Materials Science and Materials Engineering. / Ed. by Saleem Hashmi. Oxford: Elsevier, 2016. P. 1. doi 10.1016/B978-0-12-803581-8.03681-X

[47] Kurlov V.N., Rossolenko S.N., Abrosimov N.V., Lebbou Kh. // Crystal Growth Processes Based on Capillarity: Czochralski, Floating Zone, Shaping and Crucible Techniques. / Ed. by Duffar Th. Chippenham, Wiltshire, UK: John Wiley \& Sons, 2010. P. 277. doi 10.1002/9781444320237.ch5

[48] Antonov P.I., Kurlov V.N. // Crystallogr. Rep. 2002. V. 47. Suppl. 1. P. S43. doi 10.1134/1.1529958

[49] Abrosimov N.V., Kurlov V.N., Rossolenko S.N. // Progr. Cryst. Growth Charact. Mat. 2003. V. 46. P. 1. doi 10.1016/S09608974(03)90001-5

[50] Kurlov V.N. // J. Cryst. Growth. 1997. V. 179. N 1-2. P. 168. doi 10.1016/S0022-0248(97)00110-3

[51] Antonov P.I., Kurlov V.N. // Progr. Cryst. Growth Charact. Mat. 2002. V. 44. N 2. P. 63. doi 10.1016/S0960-8974(02)00005-0

[52] Katyba G.M., Zaytsev K.I., Dolganova I.N., Shikunova I.A., Chernomyrdin N.V., Yurchenko S.O., Komandin G.A., Reshetov I.V., Nesvizhevsky V.V., Kurlov V.N. // Progr. Cryst. Growth Charact. Mat. 2018. V. 64. N 4. P. 133. doi 10.1016/j.pcrysgrow.2018.10.002

[53] Kurlov V.N., Shikunova I.A., Ryabova A.V., Loschenov V.B. // Bull. Russ. Acad. Sci. Phys. 2009. V. 73. N 10. P. 1341. doi 10.3103/S1062873809100086

[54] Shikunova I.A., Volkov V.V., Kurlov V.N., Loschenov V.B. // Bull. Russ. Acad. Sci. Phys. 2009. V. 73. N 10. P. 1345. doi 10.3103/S106287380

[55] Shikunova I.A., Stryukov D.O., Rossolenko S.N., Kiselev A.M., Kurlov V.N. // J. Cryst. Growth. 2017. V. 457. P. 265. doi 10.1016/j.jcrysgro.2016.08.062

[56] Shikunova I.A., Dubyanskaya E.N., Kuznetsov A.A., Katyba G.M., Dolganova I.N., Mukhina E.E., Chernomyrdin N.V., Zaytsev K.I., Tuchin V.V., Kurlov V.N. // Proc. SPIE. 2018. V. 10716. P. 1071615. doi 10.1117/12.2306134

[57] Shikunova I.A., Dolganova I.N., Dubyanskaya E.N., Mukhina E.E., Zaytsev K.I., Kurlov V.N. // Proc. SPIE. 2018. V. 10716. P. 1071605. doi 10.1117/12.2316502

[58] Dubyanskaya E.N., Chernomyrdin N.V., Dolganova I.N., Kuznetsov A.A., Mukhina E.E., Safonova L.P., Donodin A.I., Shikunova I.A., Zaytsev K.I., Kurlov V.N. // Proc. SPIE. 2018. V. 10685. P. 1068529. doi: 10.1117/12.2306946

[59] Shikunova I.A., Zaytsev K.I., Stryukov D.O., Dubyanskaya E.N., Kurlov V.N. // Proc. SPIE. 2017. V. 10411. P. 104110Q. doi 10.1117/12.2286124 
[60] Shikunova I.A., Kurlov V.N. // J. Phys. Conf. Ser. 2016. V. 672. P. 012018. doi 10.1088/1742-6596/672/1/012018

[61] Coburger J., Wirtz C.R. // J. Neurooncol. 2018. V. 28. doi 10.1007/s11060-018-03052-4

[62] http://www.optofiber.ru/

[63] Rossolenko S.N., Kurlov V.N., Asrian A.A. // Cryst. Res. Technol. 2009. V. 44. N 7. P. 689. doi 10.1002/crat.200900067

[64] Rossolenko S.N., Kurlov V.N., Asrian A.A. // Cryst. Res. Technol. 2009. V. 44. N 7. P. 701. doi 10.1002/crat.200900068

[65] Kurlov V.N., Rossolenko S.N. // J. Cryst. Growth. 1997. V. 173. N 3-4. P. 417. doi 10.1016/S0022-0248(96)00836-6

[66] Manjila S., Mencattelli M., Rosa B., Price K., Fagogenis G. Dupont P.E. // Neurosurg. Focus. 2016. V. 41. N 3. P. E13. doi 10.3171/2016.7.FOCUS16181 